\title{
Thinking of Unilateral Osteitis Condensans Ilii in the Emergency Room: Case of a Young Pregnant Female with Back Pain
}

\author{
Sadaf Sheikh, Umair Javed and Muhammad Akbar Baig \\ Department of Emergency Medicine, The Aga Khan University Hospital, Karachi, Pakistan
}

\begin{abstract}
Osteitis condensans ilii $(\mathrm{OCl})$ remains an orthopedic diagnostic dilemma; moreover, such presentation in the emergency room (ER) is unique. Limited awareness about this topic compels emergency and internal medicine physicians to perform extensive investigations which often leads to misdiagnosis. A 23-year pregnant female presented in the Emergency Room with severe low back pain, who was treated conservatively with radiological evidence of $\mathrm{OCl}$. The mainstay treatments are analgesics and physiotherapy.
\end{abstract}

Key Words: Osteitis condensans ilii, Back pain, Pregnancy, Triangular sclerosis.

How to cite this article: Sheikh S, Javed U, Baig MA. Thinking of Unilateral Osteitis Condensans Ilii in the Emergency Room: Case of a Young Pregnant Female with Back Pain. J Coll Physicians Surg Pak 2020; 30(12):1343-1344.

\section{INTRODUCTION}

Osteitis condensans ilii $(\mathrm{OCl})$ is reported in females of childbearing age in pre-partum or post-partum periods. ${ }^{1}$ As a matter of fact, persistent back pain during pregnancy should alert a physician to the possibility of this entity. It is a rare and benign pathology due to increased ligamental laxity and mechanical stress across the sacroiliac joint with the sclerosis typically limited to the iliac bone. ${ }^{1}$

We, herein, report a case of a 23-year pregnant female presenting in the Emergency Room with severe low back pain, who was diagnosed and treated conservatively for this condition.

\section{CASE REPORT}

A 23-year female, previously healthy, primigravida with gestational amenorrhea of 7 months, presented to the Emergency Department with severe lower back pain more on the left side for 15 days that has progressively worsened to an unbearable extent. Pain was radiating down the left thigh, was continuous, worsened with activity and was causing difficulty in walking. Pain was marked across her lower back and left buttock and it occasionally radiated down the back of her left leg till big toe.

Correspondence to: Dr. Sadaf Sheikh, Department of Emergency Medicine, The Aga Khan University Hospital, Karachi, Pakistan

E-mail: sheikh.sadaf@gmail.com

Received: October 07, 2019; Revised: November 16, 2019; Accepted: November 19, 2019

DOI: https://doi.org/10.29271/jcpsp.2020.12.1343
There was no history of flu-like symptoms in recent past. Patient reported normal bowel habits. There was no history of skin rash. Patient denied numbness in the perineal and perianal region and had no history of urinary retention. There was no history of morning stiffness and pain in other small and large joints. She had no family history of joint pathology. She never complained of muscle wasting or weight loss.

On examination, the initial vital signs revealed temperature of $36.6^{\circ} \mathrm{C}$, pulse, $102 \mathrm{bpm}$, blood pressure, $130 / 76 \mathrm{mmHg}$, respiratory rate, $16 / \mathrm{min}$ and $\mathrm{O}_{2}$ saturation, $99 \%$ on room air. General physical examination was unremarkable. There was no visible rash, pallor, jaundice, lymphadenopathy, clubbing, koilonychia or thyroid enlargement. Chest, cardiovascular and abdominal examinations were unremarkable. Neurological examinations, including cranial nerves examination and detailed motor and sensory examination (power, tone, reflexes and sensation), were unremarkable but revealed positive straight leg raised test on left side. She was able to walk on her toes. Musculoskeletal examination showed normal flexion, extension, rotation and lateral bending of the lumbar spine. Palpation revealed local tenderness over both sacroiliac joints, more marked on the left side. There was no local erythema or warmth. Sciatic stretch test, femoral stretch test, Faber and Mennel tests were negative. Modified Schober test were unremarkable.

Initial laboratory workup (Table I) showed anti-double stranded (DS) DNA titre to be normal $(2.52 \mathrm{IU} / \mathrm{ml}$ - negative below 20 $\mathrm{IU} / \mathrm{ml}$ ). Antinuclear antibody was also negative. However, complement C3 and C4 levels were slightly high: C3, $2.45 \mathrm{~g} / \mathrm{L}$ and $\mathrm{C} 4,0.43 \mathrm{~g} / \mathrm{L}$.

Ultrasound pelvis revealed single alive intrauterine fetus, corresponding to 28 weeks and 5 days of gestation. Magnetic reso- 
nance imaging showed abnormal hyper-intense signals in the iliac part of left sacroiliac joint on stir sequences, suggestive of $\mathrm{OCI}$. Focal T2/FLAIR hyper-intense signals were appreciated at the region of left obturator externus muscle likely representing muscle oedema. The bilateral hip and sacroiliac joints are grossly unremarkable with no evidence of joint effusion.

Table I: Laboratory parameters.

\begin{tabular}{|l|c|}
\hline Hemoglobin (g/dL) & 10.5 \\
\hline Hematocrit (\%) & 34 \\
\hline Mean corpuscular volume (fL) & 80.4 \\
\hline White blood cells (cells per cubic millimeter of blood) & 10.7 \\
\hline Neutrophils (109 cells/liter) & 71.9 \\
\hline Lymphocytes (109 cells/liter) & 22.6 \\
\hline Platelets (109 cells/liter) & 280 \\
\hline Total bilirubin (mg/dl) & 0.5 \\
\hline Indirect bilirubin (mg/dL) & 0.2 \\
\hline Direct bilirubin (mg/dL) & 0.3 \\
\hline Gamma glutamate transferase (U/L) & 38 \\
\hline Alanine aminotransferase (U/L) & 43 \\
\hline Aspartate aminotransferase (U/L) & 51 \\
\hline Alkaline Phosphatase (IU/L) & 155 \\
\hline Erythrocyte sedimentation rate (mm) & 110 \\
\hline C-reactive protein (mg/L) & 7.42 \\
\hline Creatine kinase (muscle/brain) (units per liter) & 12 \\
\hline Random glucose (mg/dl) & 91 \\
\hline Blood cultures & No growth \\
\hline Urine cultures & No growth \\
\hline
\end{tabular}

Patient was admitted for pain management. During the course, patient was managed with intravenous paracetamol, intermittent dose of nalbuphine; but due to severe pain, patient was started on nalbuphine infusion. Acute pain management service (APMS) was also consulted. Pain gradually improved and pain medications were optimised, accordingly. During its course, she delivered a healthy baby. On 8th day of admission, pain became bearable while being on oral tramadol, paracetamol and pregabalin; and patient was discharged on oral analgesics.

\section{DISCUSSION}

Barsony and Polgar first described the increased condensation of the ilium in 1928 and they suggested the term OCl to designate this condition. They also reported 15 cases in which iliac changes were noticed and demonstrated radiographically. ${ }^{1}$

$\mathrm{OCl}$ is a radiological diagnosis on plain X-ray featured by sclerosis of iliac bone adjacent to normal sacroiliac joint causing back and hip pain. Mechanical stress is mostly thought as contributing factor for this benign pathology. Bilateral sacroiliac joints are involved. Literature supports its bilateral involvement but in our case, it was unilateral.

The changes, which are restricted to the ilium, are not extensive and never involve the sacrum or any other bone. Most authors mentioned mostly three predisposing factors: female gender, young age, pregnancy and childbirth.

In practice, this condition can be potentially confused with seronegative spondyloarthropathy, metastatic disease or sacroilitis.

Making a diagnosis of $\mathrm{OCl}$ in pregnant population requires awareness of this condition and radiological findings emphasising on triangular sclerosis on the iliac aspect of the sacroiliac joint with preserved joint space. ${ }^{2}$ Low back pain, as described in our case, is the typical presenting feature: it is slow and gradual with radiation to gluteal region and posterior aspect of the thighs with positive Faber's test. ${ }^{3}$ Treatment options are physiotherapy and analgesia. It has a self-limiting course. Literature shows that a small group of patients may develop persistent pain secondary to $\mathrm{OCl}$. For refractory cases, surgical resection and arthrodesis are procedures offered with no guaranteed success. A novel mini-invasive surgical approach through percutaneous iliac core decompressions through a cannulated drill bit was studied by Ayoub MA for refractory cases. ${ }^{4}$ It showed great benefit for refractory $\mathrm{OCl}$ cases with almost no added morbidity or complications. It had the advantage of sparing the physiological functions of the affected sacroiliac joints.

$\mathrm{OCl}$ presentation in the Emergency Department is unique and should altert an emergency physician to help differentiate it from other mimics. It is imperative to think of this and establish this diagnosis promptly to avoid extensive investigations.

\section{PATIENT'S CONSENT:}

Verbal consent was taken from the patient.

\section{CONFLICT OF INTEREST:}

The authors declared no conflict of interest.

\section{AUTHORS' CONTRIBUTION:}

SS, UJ, MAB: All authors equally contributed in the development and completion of this manuscript.

\section{REFERENCES}

1. Mitra R. Osteitis condensans ilii. Rheumatol Int 2010; 30:293.

2. Çidem M, Capkin E, Karkucak M. Osteitis condensans ilii in differential diagnosis of patients with chronic low back pain: A review of the literature. Mod Rheumatol 2012; 22(3): 467-9. doi: 10.1007/s10165-011-0513-9.

3. Cicone C, Giordano N, Kural B, Mansukhani S, Agarwal S. Poster 406 Osteitis Condensans IIli $(\mathrm{OCl})$, a rare cause of low back pain: A case report. P M R 2016; 8(9S):S294. doi: 10.1016/j.pmrj.2016.07.333.

4. Ayoub MA. Refractory osteitis condensans ilii: Outcome of a novel mini-invasive surgical approach. Int Orthop 2013; 37(7):1251-6. doi: 10.1007/s00264-013-1901-x. 